\title{
19
}

\section{Current Issues on Modeling Extreme Inflows in Stormwater Systems}

\author{
Jose G. Vasconcelos, Steven J. Wright and Philip L. Roe
}

The increasing availability of different numerical models to simulate flow in storm sewers has raised several questions on the applicability of these models in the simulation of extreme inflow conditions. These issues include accurate modeling and prediction of bores, flow regime transition into pressurized flow and the possibility of air pocket entrapment and the subsequent effect on the system.

With regards to bore description, the issue is not limited to the choice of equations for the problem solution, but includes the selection of numerical schemes that will ensure the correct bore propagation speed. It will be shown that the selection of the variables used to solve the mass and momentum equations has an impact in the solution of discontinuous flows. It will also be seen that numerical models without shock-capturing capabilities have limited ability to model flow discontinuities.

Flow regime transition models can be divided into shock-fitting or shock-capturing approaches, each group of approaches with particular strengths and limitations. Due to the popularity of Preissmann slot-based models, including the soon-to-be released SWMM 5, a more detailed discussion on the issues of this approach is provided. Some new model improvements to shock-capturing approaches are presented, including a new conceptual model for simulating sewer surcharging referred to as the Decoupled Pressure Approach.

Vasconcelos, J., S.J. Wright and P.L. Roe. 2006. "Current Issues on Modeling Extreme Inflows in Stormwater Systems." Journal of Water Management Modeling R225-19. doi: 10.14796/JWMM.R225-19.

(C) CHI 2006 www.chijournal.org ISSN: 2292-6062 (Formerly in Intelligent Modeling of Urban Water Systems. ISBN: 0-9736716-2-9) 
Finally, some of the current challenges in the incorporation of the air phase in the computation of the rapid filling pipe problem are considered. These challenges include the limitations of the one-dimensional framework, difficulties of dealing with boundary conditions for the air phase and the possibility of incorporating the air phase in either a two-phase flow or a submodel framework.

\subsection{Introduction}

The development of computational models has improved the ability to describe flow in stormwater sewers. Different model frameworks are currently available to describe transient flows in sewers. These models usually assume that the pressure distribution is hydrostatic and characterized by long waves with little variation in the transversal direction. These assumptions are reasonable for flows in stormwater sewers, and the integration of the mass and momentum equations in an arbitrary control volume yield the so-called divergence form of the equations governing open-channel flows in sewers (for prismatic channels with no lateral inflow):

$$
\begin{aligned}
& \frac{\partial A}{\partial t}+\frac{\partial Q}{\partial x}=0 \\
& \frac{\partial Q}{\partial t}+\frac{\partial}{\partial x}\left(\frac{Q^{2}}{A}+g A h_{c}\right)=g A\left(S_{o}-S_{f}\right)
\end{aligned}
$$

In the above equations, $A$ is the flow area, $Q$ is the inflow rate, $h_{c}$ is the centroid depth, $S_{o}$ is the bed slope and $S_{f}$ is the energy slope.

The scope of this work is to understand how the models based on these equations perform while simulating extreme inflow conditions in stormwater systems. These inflows, expected during intense rain events, may result in bore formation, transition from open-channel flow into pressurized flow, and air pocket entrapment and pressurization. As will be shown, there are particular issues that have to be considered in such cases to assure the accuracy of the computations.

Initially, a discussion regarding the appropriate selection of variables to solve the mass and momentum equations is presented along with the type of numerical schemes that are appropriate to model the bore propagation in stormwater sewers. Following this, a discussion on the available flow regime 
transition models is presented and their applicability is assessed. An alternative to the traditional Preissmann slot concept, here called the Decoupled Pressure Approach (DPA), is outlined. Finally, the current challenges of incorporating air phase in the sewer flow problem are presented, along with some ideas that can indicate how current models can be improved in this aspect.

\subsection{Selection of Appropriate Variables and Numerical Schemes}

\subsubsection{Variable Selection}

Several numerical models are widely available to simulate sewer flows employing Equations 19.1 and 19.2, such as SWMM (EPA, 2004), MOUSE (DHI, 2003), ISS (Sevuk et al., 1973), among others. It is possible to rewrite these equations using depth and velocity as the conserved variables. Then, the mass and momentum equations become:

$$
\begin{aligned}
& \frac{\partial y}{\partial t}+V \frac{\partial y}{\partial x}+\frac{A}{B} \frac{\partial V}{\partial x}=0 \\
& \frac{\partial V}{\partial t}+V \frac{\partial V}{\partial x}+g \frac{\partial y}{\partial x}=g\left(S_{o}-S_{f}\right)
\end{aligned}
$$

In the above equations, $y$ is the flow depth, $V$ is the velocity, and $B$ is the width of the free-surface flow. While the above equations are an equivalent representation of the mass and momentum equations, depth and velocity are not physically meaningful conserved variables in sewer flows (Toro, 2001).

Issues with applying Equations 19.3 and 19.4 instead of Equations 19.1 and 19.2 are clearly noticed when flow discontinuities are present in the simulation. Across flow discontinuities, spatial derivatives $(\partial / \partial x)$ are not defined, and integral forms of the conservation laws are required. When the selected variables are the flow area and discharge $\left(\mathbf{U}=[A, Q]^{\mathrm{T}}\right)$, the integration of the mass and momentum equations at the vicinity of the shock results in the Rankine-Hugoniot conditions (Katopodes, 2003), which hold across the bore. Because Equations 19.1 and 19.2 satisfy the differential forms on either side of the bore and the Rankine-Hugoniot conditions at the bore location, these equations represent weak solutions of the mass and momentum conservation laws for open-channel flows. This permits the 
creation of numerical schemes that handle both continuous and discontinuous flows, provided that the entropy conditions are satisfied.

On the other hand, if the choice of dependent variables is such that $\mathbf{U}=$ $[y, V]^{\mathrm{T}}$, then simulated bores have incorrect propagation speed. Figure 19.1 illustrates this issue in an arbitrary problem where a bore is generated in a 1$\mathrm{m}$ diameter, 100-m long, horizontal pipe, with Manning rugosity of 0.010 . There is an initial amount of water in the pipe (either $0.30 \mathrm{~m}$ or $0.40 \mathrm{~m}$ of water depth), and no flow conditions. At the upstream end, the water level is instantaneously raised to $0.60 \mathrm{~m}$ at $\mathrm{T}=0 \mathrm{~s}$, generating thus the bore.
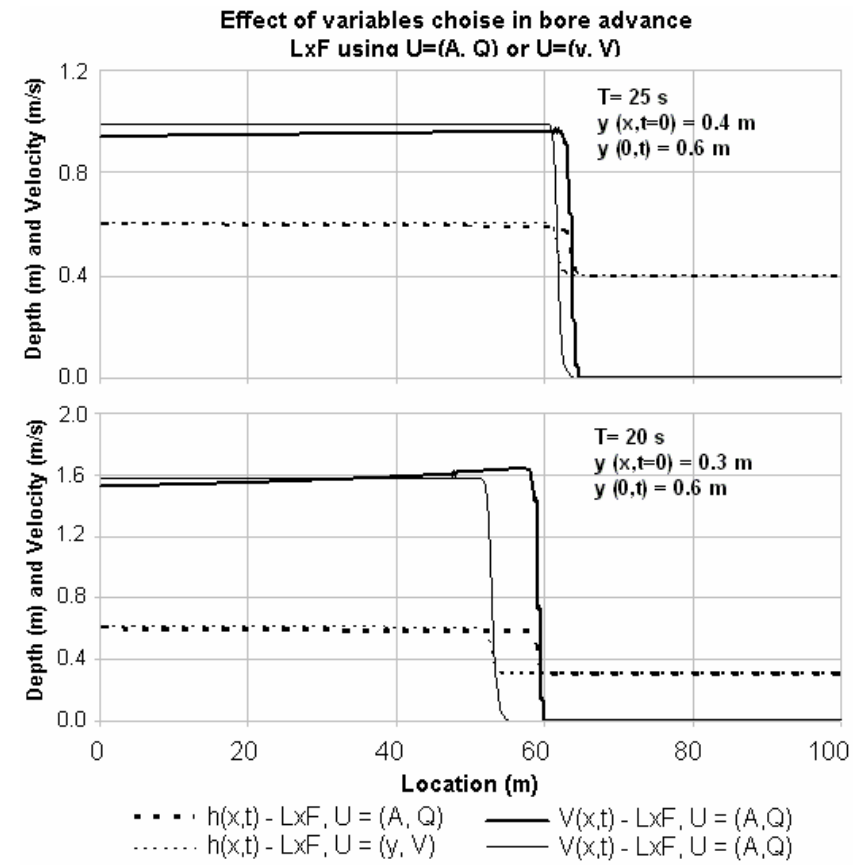

Figure 19.1 Bore propagation results for two initial water depths using different set of equations to solve the flow.

Figure 19.1 shows the depths and velocity profiles obtained by applying either Equations 19.1 and 19.2 (using the variables vector $\mathbf{U}=[A, Q]^{\mathrm{T}}$ ) or Equations 19.3 and 19.4 (using the variables vector $\mathbf{U}=[y, V]^{\mathrm{T}}$ ) to solve the mass and momentum equations. The latter choice yields a bore with slower propagation speeds; this discrepancy is more noticeable for larger depth discontinuities across the bore. These results are in agreement with Toro 
(2001), who predicted this behavior for the one-dimensional, shallow water equations. All problems where solved using the Lax-Friedrich scheme $(\mathrm{LxF})$, with maximum Courant number of 0.9 .

\subsubsection{Numerical Scheme Selection}

With regard to the numerical schemes to solve the transient flow equations during extreme inflow conditions, accurate bore propagation can be achieved with shock-capturing methods. Numerical schemes such as the Lax-Friedrichs, Lax-Wendroff and Roe $1^{\text {st }}$ order upwind (Roe, 1981), when applied to Equations 19.1 and 19.2, are able to model the formation and propagation of open-channel bores with reasonable accuracy. The advantage of these schemes is that a single scheme is used throughout the problem domain. The main drawback is that some numerical diffusion is present in the predicted bore, particularly for linear, $1^{\text {st }}$ order accurate schemes. Nonetheless, because of the overall simplicity and relatively low computational costs, such schemes became popular.

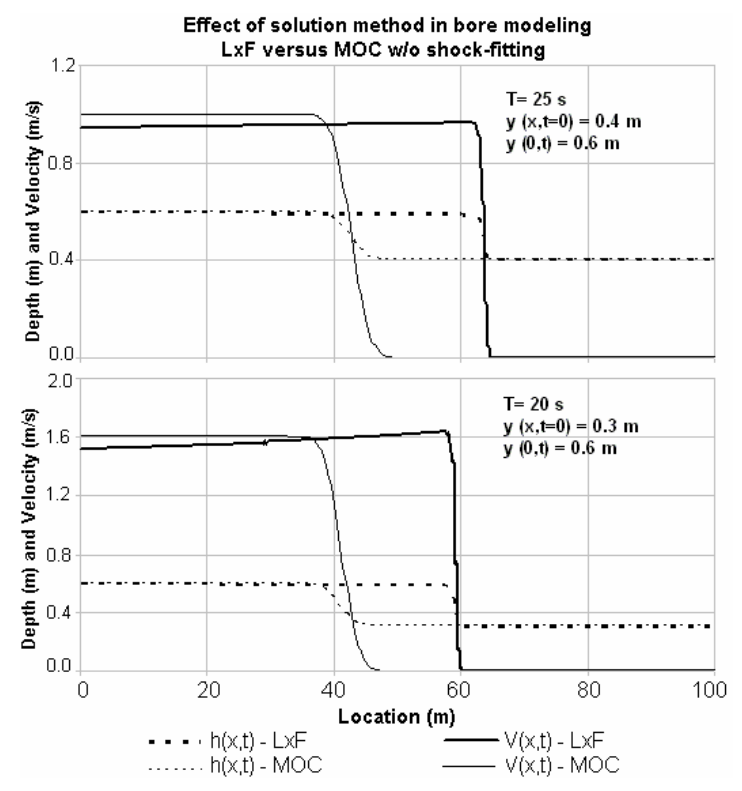

Figure 19.2 Bore propagation results for two initial water depths using either the LxF scheme or MOC without shock-fitting. 
An alternative way to model the bore propagation is to use shock-fitting schemes. A shock-fitting scheme could consist of a Method of Characteristics (MOC) solver applied on both sides of the bore, and a set of equations to be solved at the bore location to assure that mass and momentum are conserved across the bore. However, if the shock-fitting procedure is not applied and a MOC solver is used to simulate a flow with bores, poor numerical results are obtained.

Figure 19.2 shows the results of the same problem (1-m diameter, $100-\mathrm{m}$ long pipeline with sudden rise of upstream depth), but in this case a model based on Equations 19.1 and 19.2 and using $\mathrm{LxF}$ is compared to the results from a solver based on the MOC-Hartree (Sturm, 2000) without applying the shock-fitting procedure. Not only is the propagation speed of the bore predicted incorrectly, but the inflow front is also more smeared in the results obtained with the MOC solver.

A serious drawback of using shock-fitting approaches to model bores is that the discontinuity tracking procedure requires the solution of a system of non-linear equations at each time step for each bore. In cases of actual stormwater systems, in which inflow occurs in several locations simultaneously, the possibility of having multiple bores exists. In such cases, the tracking of each one of these fronts by shock-fitting models can be very expensive in terms of computational effort. It is also possible that special measures are needed to handle cases when bore collides. This issue is also present when flow regime transition models are considered, and this is discussed in the following section.

\subsection{Model Approaches for Flow Regime Transition}

Some extreme inflows may result in the transition from free-surface flow into a pressurized flow regime. This occurrence, due to conveyance limitation of the sewers as open-channels, is referred in the literature as flow regime transition or mixed-flow. Special procedures to model such conditions are required since the open-channel mass and momentum equations are not valid for pressurized flow conditions.

Generally, flow regime transition fronts can be viewed as a special type of sewer bore. In this sense, the model approaches for the flow regime transition problem are also classified as shock-capturing and shock-fitting approaches. However, it is possible that a gradual transition between openchannel flow and pressurized flow occur in a location far away from the 
bore. The possibility of such "gradual" transitions is among the reasons why shock-capturing models are preferred over shock-fitting models.

The different types of flow regime transition models based on shockfitting approaches depend on the solution procedure employed in the pressurized and free surface portions of the flow. Many models solve the pressurized portion of the flow assuming rigid column behavior, the socalled lumped inertia approach (Wylie and Streeter, 1993). These types of models include the approach presented by Wiggert (1972) that solves the free surface portion of the flow using the MOC. The model proposed by $\mathrm{Li}$ and McCorquodale (1999) uses the lumped inertia approach for both the free surface and the pressurized portions of the flow. The model proposed by Zhou et al. (2002) assumes that the flow regime transition front is characterized by a vertical interface that advances in a pipe filled with air, which can become pressurized as it is expelled from the pipe by the inflow bore.

While conceptually simple, shock-fitting models based on the rigid column approach have severe limitations with regards to their applicability. First, if the inflow bore has insufficient strength to close the pipe crosssection, some other model is required to simulate the flow. Second, these models cannot be applied if the flow regime transition occurs in a location different from the inflow bore. Finally, the models cannot simulate complex flow configurations such as the Interface Reversal (Cardle and Song, 1988) and the Interface Breakdown (Vasconcelos and Wright, 2005a).

A more capable type of shock-fitting model is represented by the fulldynamic approach used by Song et al. (1983), Cardle and Song (1988), Guo and Song (1990), among others. The main difference is that such models use the MOC both in the pressurized and free-surface portions of the flow. This confers more flexibility to simulate extreme inflow conditions. Yet, shockfitting models have the drawback of needing to track the bores, which can be too expensive in terms of computational effort. And the issue of having the flow regime transition occurrence not coincident with the inflow front also challenges full-dynamic model assumptions. These issues are not shared by flow regime transition models based on shock-capturing methods; most of these models are constructed with the Preissmann slot concept. The strengths and limitations of such models are discussed in the following section. 


\subsection{Preissmann Slot Models: Strengths and Limitations}

As explained, shock-capturing models use the same numerical scheme to solve the flow throughout the domain when bores are present. When the free-surface conditions are present in the whole domain, the application of such models in sewer flows is straightforward. Examples of shock capturing models used to simulate open channel flows are provided by Glaister (1988), Garcia-Navarro et al. (1994), and others.

The issue becomes more complex when the water level reaches the pipe crown resulting in flow pressurization since Equations 19.1 and 19.2 become undefined. An ingenuous solution was presented in the work by Cunge and Wegner (1964), following a previous suggestion by Preissmann to simulate a hypothetical slot on the top of the pipe. This slot objectives are: (i) allow the surcharging of the pipes without need to change equations and (ii) allow for the much faster pressure wave celerities required in the pressurized portion of the flow.

The slot allows the centroid depth $h_{c}$ to exceed half of the pipe diameter $\mathrm{D} / 2$. This in turn allows for the existence of the additional pressure force that results from the pipe pressurization. The slot width is very small because it has to be sized to match the expected celerity of the waves in the pressurized portion of the flow according to the relation for free surface wave speed:

$$
T_{\text {Slot }}=\frac{g A}{a^{2}}
$$

In the above equation, $T_{\text {slot }}$ is the width of the hypothetical slot, and $a$ is the celerity of the acoustic wave is the pressurized portion of the flow. The value of acoustic wave-speeds in actual systems is hard to estimate because of its strong dependency on the air content of the water, which itself is unknown. Nevertheless, it is expected that the pressurized flow acoustic wave-speed in sewers is on the order of hundreds of meters per second or more.

The introduction of the Preissmann slot in the problem transforms the flow regime transition problem into a standard free-surface flow problem for a channel with a peculiar cross-sectional shape. This simple concept was the main reason for the popularization of this approach, implemented in models such as the MOUSE (DHI, 2003), SWMM 5 (EPA, 2004), and others. 
There are, however, some limitations of flow regime transition models constructed with the Preissmann slot concept:

1. If the slot is selected to yield acoustic wave-speeds on the order of hundreds of meter per second, the resulting time step in the computations is excessively small. Thus, larger slot widths are normally chosen, despite the fact that the resulting acoustic wave-speed are unrealistically small; this has an effect on the accuracy of the solution as explained below.

2. When the flow regime transition is coincident with the bore location, spurious numerical oscillations appear in the solutions. These have been reported by several authors, e.g. Trajkovic et al. (1999) and Vasconcelos et al. (2005). The causes of these oscillations have been explained by Arora and Roe (1997).

3. Preissmann slot models cannot simulate pressurized flows with a piezometric heads below the pipe crown, as pointed out by Cunge et al. (1980) and Song et al. (1983). If, in a pressurized portion of the flow, the piezometric head drops below the pipe crown, the free surface is regenerated in these models. However, since this regeneration is only possible if ventilation is available at that location, this limitation may compromise the accuracy of such models.

\subsubsection{Impact of the Slot Width in the Flow Regime Transition Simulation}

In addition to the intended effects during pressurization events, the introduction of the Preissmann slot has an impact on the storage capacity of the pipe cross section. The slot introduction increases the cross sectional flow area by the slot width times the surcharge pressure head. Even if the slot width is considered narrow, a large surcharge head can result in significant water storage in the slot during the pressurization event, influencing the accuracy of the computations.

To illustrate the impact of the assumed slot width on the computations, an example is described: in a 1-m diameter, 1000-m long, horizontal pipe, with Manning rugosity of 0.010 , the water level is initially at $0.75 \mathrm{~m}$, and a 
no-flow condition exists in the system. At the upstream end, the water level is instantaneously raised to $4.0 \mathrm{~m}$ at $\mathrm{T}=0 \mathrm{~s}$, and the resulting pipe-filling bore will initiate flow regime transition. A Preissmann slot based model is used to simulate the flow. This model uses the Roe $1^{\text {st }}$ order upwind scheme, maximum Courant number of 0.95 , a 400 -cell grid and simulates the initial 70 seconds of the flow using different assumed values for the acoustic wavespeed in the pressurized side of the bore. The simulation returned the location of the inflow front for each one of the cases. The results are presented in Table 19.1 and Figure 19.3.

Table 19.1 Flow simulation results obtained by a Preissmann slot model for different assumed acoustic wave-speeds.

\begin{tabular}{ccccc}
\hline$a(\mathrm{~m} / \mathrm{s})$ & $T_{\text {slot }}(\mathrm{m}) / \mathrm{D}$ & $\begin{array}{c}\text { Inflow volume } \\
\text { admitted }\left(\mathrm{m}^{3}\right)\end{array}$ & $\begin{array}{c}\text { Inflow volume } \\
\text { stored in slot (\%) }\end{array}$ & $\begin{array}{c}\text { Error in the front location } \\
\text { at } \mathrm{T}=70 \mathrm{~s}(\%)\end{array}$ \\
\hline 12.5 & $4.93 \mathrm{E}-02$ & 195.50 & 40.87 & 10.06 \\
18 & $2.38 \mathrm{E}-02$ & 160.95 & 25.21 & 6.12 \\
25 & $1.23 \mathrm{E}-02$ & 145.64 & 14.93 & 3.39 \\
50 & $3.08 \mathrm{E}-03$ & 133.21 & 4.21 & 0.67 \\
100 & $7.70 \mathrm{E}-04$ & 129.81 & 1.09 & 0.06 \\
200 & $1.93 \mathrm{E}-04$ & 128.70 & 0.27 & 0.00 \\
\hline
\end{tabular}

As Table 19.1 indicates, wider slots may store a significant portion of the inflow volume. Since larger amounts of water are stored at the slot, the advance of the inflow front is smaller in such cases, and in this particular example, the error can be over $10 \%$ for the initial $70 \mathrm{~s}$ of simulation. Figure 19.3 shows the results when the relative error of front location is plotted against the assumed pressurized acoustic wave-speed and the relative inflow volume stored at the slot. The front location error decreases with an inverse power law of the assumed acoustic wave-speed. There is a direct, quasilinear relation between the inflow front location error and the relative inflow volume of water stored in the slot. This clearly indicates the impact of the slot width on the calculation accuracy.

Of course, the longer the tunnel, the larger will be the absolute error in the inflow front location, since the bore speed is not correct. However, the relative error is approximately the same when the calculations are repeated assuming the pipe length of $2000 \mathrm{~m}$ for $200 \mathrm{~s}$ simulations. The relative error is increased when the assumed constant pressure head at the upstream end 
increases from $4 \mathrm{~m}$ to $6 \mathrm{~m}$, showing that for large pressurization conditions, more water is stored in the slot, causing a decrease in the model accuracy.

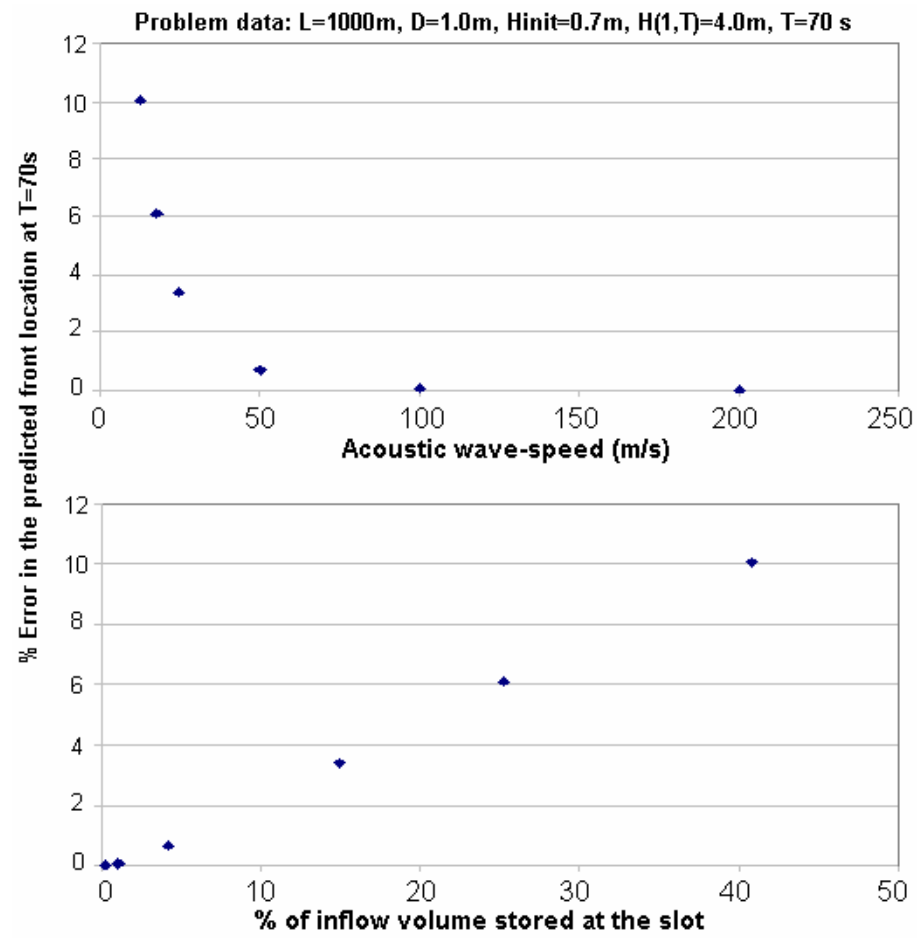

Figure 19.3 Relative error in the calculation of the advance of the inflow front for $\mathrm{T}=70 \mathrm{~s}$ for different acoustic wave-speeds using the Preissmann slot model.

If pressurized conditions are expected to last for a significant amount of time, the width of the slot should be selected to reproduce the correct celerity for transient pressure waves in the pressurized portion of the flow. Failure to adopt appropriate slot widths may also compromise the modeling of events such as pump start-up of a totally filled below grade storage tunnel.

In summary, the location of the advancing inflow front obtained by Preissmann slot models simulation depends on the assumed width of the slot. This width, if too wide, will underestimate the actual advance of the 
inflow front, which in some cases may compromise the overall accuracy of the model.

\subsubsection{Numerical Oscillations in Flow Regime Transition Simulations}

Flow regime transition fronts may be characterized by a sharp discontinuity in the celerity and the pressure head across the pipe-filling bore. These are extreme non-linear conditions, and as a result, Preissmann slot model simulations show spurious numerical oscillations on the pressurized side of the bore. These post-shock oscillations are explained in the work by Arora and Roe (1997), and have been acknowledged by different authors such as Trajkovic et al. (1999) and Vasconcelos et al. (2005).

These post-shock oscillations are caused by the solution procedure that shock-capturing models use to model discontinuities, and to date, there is no adequate solution for the issue. Some approaches to alleviate the problem are: use of wider slots (thus reducing differences in the celerities across the bore); and use of diffusive numerical schemes. As mentioned previously, wider slots have an impact on the model accuracy. Diffusive numerical schemes, such as Lax-Friedrichs, are inappropriate for flow regime transition simulations because of the poor results in the free surface portion of the flow (Vasconcelos and Wright, 2004), because of the extremely low Courant number in that region.

If the value used for the acoustic wave-speed is small, these oscillations have a reduced effect on the model accuracy. As the assumed wave-speed increases, the stability of a Preissmann slot model can be compromised by the oscillations. That is caused by the oscillations in the modeled pressure head, which can become negative, causing the program to crash.

A new alternative to mitigate the problem was proposed by Vasconcelos and Wright (2005b) by applying a numerical filter to the modeled results. This filter is designed to reduce the amplitude of the oscillations, and is based on a Fast-Fourier Transform procedure (FFT), as suggested by Press et al. (1989). Figure 19.4 illustrates the case for the problem posed in subsection 19.4.1 when $\mathrm{c}=100 \mathrm{~m} / \mathrm{s}$. The thinner line is the unfiltered results of the pressure head profile at $\mathrm{T}=70 \mathrm{~s}$. The thick line shows the results of the same simulation after the FFT filter is applied, and a significant reduction in the magnitudes of the numerical oscillations is observed. However, the filtering requires additional calculations that can increase the computational effort by up to $60 \%$. 


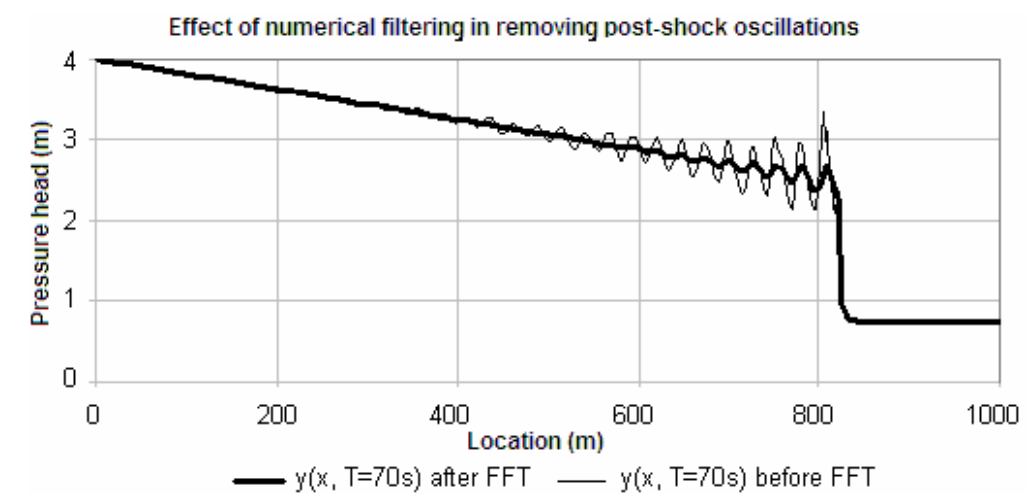

Figure 19.4 Effect of a Fast-Fourier Transform filtering in eliminating numerical oscillations from the results.

\subsection{Preissmann Slot Alternative: Decoupled Pressure Approach}

The simplicity of the Preissmann slot concept explains in part why this approach became so popular for flow regime transition models. Some of the issues discussed in the previous section are normally addressed by using low values for the acoustic wave-speed, despite the resulting loss of the model accuracy and of the impaired ability to simulate extended periods of pressurized, transient flows in the system.

There is, however, another significant problem with the Preissmann slot concept: the inability of this approach to simulate pressurizes flows with the piezometric head below the pipe crown. As in the situation of a siphon, pressurized flows can be sustained in low and even negative piezometric heads. However, if a model based on the Preissmann slot concept is used to simulate such flows, the free surface interface would be regenerated. This, of course, can only occur if ventilation is available at that location, otherwise this regeneration has no physical basis.

The source of this problem is in the way Preissmann slot models handle the over-pressurization caused by the surcharge flows. If a surcharge occurs, the additional head is stored in the slot, and thus the only term affected is the one related to the centroid depth, as Equation 19.2 indicates. The pressure term related to the presence of the water in the cross section 
(hydrostatic-like) and the surcharge pressure term are treated together, which is adequate for surcharging flows where both terms are positive. However, if the surcharge pressure term becomes negative, the sum of the pressure head terms becomes less than the pipe depth, causing the regeneration of the free surface.

The Preissmann slot is, of course, just a conceptual device to allow overpressure to be generated when the pipe is running full, and the failure just described comes from taking it too literally. One solution to this problem is to find an alternative way of calculating an overpressure. As suggested by Vasconcelos et al. (2005), the pressure in the momentum Equation 19.2 can be split into two terms:

$$
\frac{\partial Q}{\partial t}+\frac{\partial}{\partial x}\left(\frac{Q^{2}}{A}+g A\left(h_{c}+h_{s}\right)\right)=g A\left(S_{o}-S_{f}\right)
$$

In the above equation, $h_{c}$ is the hydrostatic-like pressure, which never exceeds the centroid depth of the pipe, or $\mathrm{D} / 2$, and an overpressure $h_{s}$. This overpressure would normally be positive but could be allowed to become negative to deal with the case where a full pipe experiences pressure drop because of a expansion wave, but no ventilation is available to regenerate a free surface.

The inspiration for our approach is to imagine that the pipe is elastic. If it were, the acoustic wave-speed would be given by (Wylie and Streeter, 1993):

$$
a=\sqrt{\frac{A}{\rho} \frac{\Delta p}{\Delta A}}
$$

and the area change $\Delta A$ would be related to the pressure rise $\Delta p$ by the elastic properties and the geometry of the pipe. Here, however, just as with the Preissmann slot, our objective is simply to match the computed celerity with some estimated celerity. So, writing $\Delta p=\rho g h_{s}$, we have:

$$
h_{s}=\frac{a^{2}}{g} \frac{\Delta A}{A}
$$


Where $a$ is the estimated celerity in the pressurized regime, and $\triangle A / A$ is calculated from the output of the continuity equation (the inflow area $A$ ). This equation can be interpreted as giving the area that the water would occupy in the elastic pipe if its density remained constant. The effective area change $\Delta A / A=\left(A-A_{\text {pipe }}\right) / A$, which can be either positive or negative depending on whether the inflow area is larger or smaller than the pipe area, respectively. The algorithm to compute it is as follows.

1. If no flow regime transition occurs, $h_{s}$ is zero throughout the domain and $h_{c}$ is calculated in terms of the conserved variable $A$. The centroid depth can only assume values between 0 and $D / 2$.

2. If flow regime transition initiates at one of the system extremes, $h_{s}$ becomes positive at that location. Pressurization conditions may propagate throughout the system.

3. If a low pressure transient wave is generated (e.g. pump start-up), the algorithm checks whether at a given location there is availability of ventilation. If not, $h_{c}$ is kept at the value of $\mathrm{D} / 2$ and $h_{s}$ becomes negative. If yes, is the low pressure transient drops the pressure below the pipe crown and a free surface is regenerated.

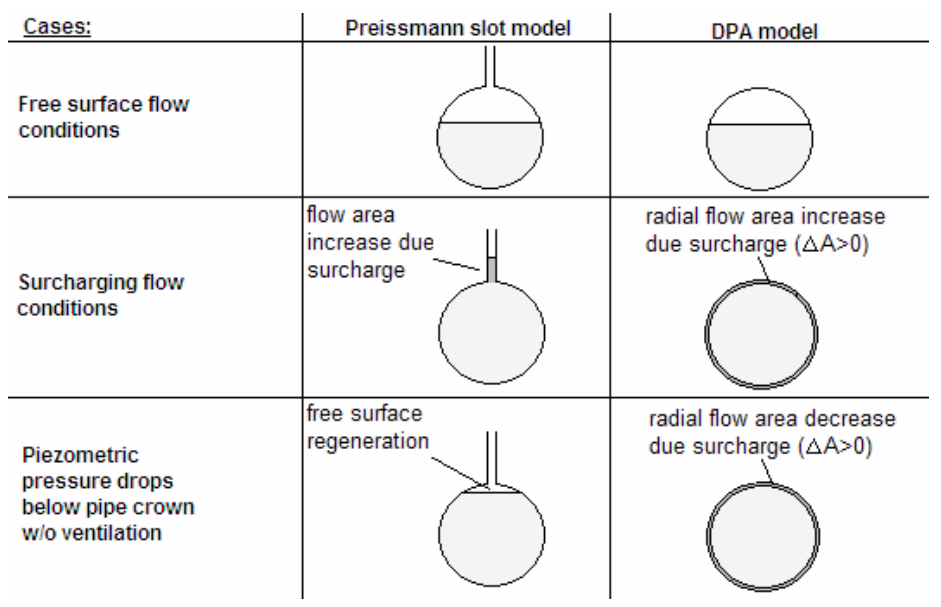

Figure 19.5 Comparative example of the Preissmann slot and DPA model behavior under different flow conditions. 
In summary, the DPA approach introduces a new term in the openchannel momentum equation that separately describes the additional pressure term expected in pressurized flow conditions. This modification retains the conceptual simplicity of the Preissmann slot models and can be added to any free-surface flow solver based on Equations 19.1 and 19.2. The resulting approach was successful in simulating both surcharge and siphon flows as well in reproducing the numerical predictions of current flow regime transition solvers. It may represent a good alternative to the Preissmann slot models.

\subsection{The Challenges of Including Air Phase in Sewer Flow Model Formulation}

The possibility of air phase entrapment and pressurization exists whenever the available ventilation in stormwater systems is inadequate. This adds considerable complexity to the sewer flow problems, and may drastically alter the liquid flow dynamics. These interactions are poorly understood to date, and they can be the source of serious operational problems.

Mild air pressurization can reduce the propagation speed of pipe-filling bores during flow regime transition events, causing an "air cushioning" effect for the pipe-filling bore within the pipe. Air phase pressurization can also cause the motion of the water phase underneath the air pocket, referred to as Pre-bore Motion by Vasconcelos and Wright (2003). In poorer ventilation conditions, air may intrude on the top of pipe-filling bores, which was referred by Vasconcelos and Wright (2005a) as Interface Breakdown. Finally, entrapped air pockets within the pressurized portion of the water flow can cause increased pressure peaks and geysering (release of air-water mixtures) through vertical shafts.

Attempts to include air phase effects in flow regime transition formulations are exemplified by the works of Hamam and McCorquodale (1982), Li and McCorquodale (1999), Nguyen (1999), Vasconcelos and Wright (2005a), among others. The scope of air/water phase interactions described by such works is limited, and includes the air cushioning effects and the "pre-bore motion". The work by Vasconcelos and Wright (2005a) explored the application limits of one-dimensional, single-phase flow models to describe flow regime transition with air phase pressurization.

A more appropriate treatment for the problem would be provided by a one-dimensional, two-phase flow formulation. In such cases, mass and 
momentum equations are considered for both water and air phases; examples of this type of model are provided by DeHenau and Raithby (1995), Arai and Yamamoto (2003), Issa and Kempf (2003), among others. The system of partial differential equations for this type of model would be:

$$
\begin{aligned}
& \frac{\partial \rho_{w} A_{w}}{\partial t}+\frac{\partial \rho_{w} Q_{w}}{\partial x}=0 \\
& \frac{\partial \rho_{a} A_{a}}{\partial t}+\frac{\partial \rho_{a} Q_{a}}{\partial x}=0 \\
& \frac{\partial Q_{w}}{\partial t}+\frac{\partial}{\partial x}\left(\frac{Q_{w}^{2}}{A_{w}}+g A_{w}\left(h_{c, w}+h_{s}+h_{\text {air }}\right)\right)=g A_{w}\left(S_{o}-S_{f, w}\right)+F_{\text {int }} \\
& \frac{\partial Q_{a}}{\partial t}+\frac{\partial}{\partial x}\left(\frac{Q_{a}^{2}}{A_{a}}+g A_{a}\left(h_{c, a}+h_{\text {air }}\right)\right)=g A_{a}\left(S_{o}-S_{f, a}\right)-F_{\text {int }}
\end{aligned}
$$

In the above equations, the subscripts $w$ and $a$ stands for water and air phases respectively, and $F_{\text {int }}$ is the shear force component between the phases, resulting from the relative motion between them. Notice that if $h_{\text {air }}$ is positive, $h_{s}$ is necessarily zero because the presence of the air phase in the section. Also, the sum $A_{w}+A_{a}$ equals the pipe cross sectional area, and $h_{\text {air }}$ and $\rho_{a}$ can be related assuming either isothermal or adiabatic compression/expansion behavior of the gas. With closure relations to determine the energy slopes for both phases and the shear stresses caused by the relative motion between them, there are only four remaining variables that are solved by the system of partial differential equations: $A_{w}, \rho_{a}, Q_{w}$ and $Q_{a}$.

A solution procedure to this problem could be based on an explicit, finite volume solver, with shock capturing capabilities. However, because of the complexity of the equations, the characteristic analysis yields no closed form for the eigenvalues and eigenvectors, restricting the use of upwind schemes. Centered schemes such as Lax-Friedrichs have been tried so far, but the numerical diffusion is too problematic. Future investigations are pointed toward the use of less diffuse schemes, such as the HLLL scheme proposed by Linde (2002).

Current two-phase flow models are widely used in gas/oil pipeline simulations, and in such applications, the usual type of boundary condition is the steady inflow of both phases at the upstream end, while at the downstream end the system is open to the atmospheric pressure. Those 
boundary conditions are very different from the ones encountered in stormwater systems, where the water inflows are unsteady and nothing can prevent air pockets to move in either the upstream or downstream direction. It is uncertain to date how the boundary condition formulation would be developed in pipes where the air phase is present only in parts of the length, such as in pockets. Finally, the numerical description of the air pocket expulsion in the sewer extremes (such as manholes) could be a complex task in such models.

\subsection{Conclusions}

The modeling of extreme inflow conditions in stormwater systems remains a major challenge for engineers. Those inflow conditions subject the drainage systems to major structural efforts caused by water-hammer like pressure peaks, sudden air pocket expulsion, geysering, and hydraulic bore impacts. To provide proper design of these structures against these events the accurate modeling of the flow conditions is fundamental.

Among the several options now available in terms of numerical models and algorithm to solve such flows, careful choice of variables and numerical schemes to be applied is required. To model accurately flow discontinuities, or bores, the mass and momentum equations need to be in divergence form, with the resulting dependent variable vector $\mathbf{U}=[\mathrm{A}, \mathrm{Q}]^{\mathrm{T}}$. Any other selection of the dependent variable will result in bores with the wrong propagation speed, compromising the model accuracy.

The correct bore modeling also depends on the appropriate selection of the numerical scheme. Currently, different shock-fitting and shock-capturing models are available, with the latter one being favored because of the overall simplicity to handle bore formation and propagation. To simulate the transition between free-surface flow regimes, shock-capturing models require a conceptual model, such as the Preissmann slot, so that the freesurface mass and momentum equations can also be applied to pressurized flow portions of the system.

The Preissmann slot model is currently used by a number of different stormwater flow solvers. While the introduction of the slot allows for the free-surface flow equations to simulate pressurized conditions, the required slot width to simulate the correct acoustic wave-speed in the pressurized portion of the flow generates time steps that are often too small for extended period simulations. While the current practice is to make the hypothetical 
slots wider to increase the model time step, this work demonstrated the adverse impact that this practice has in the correct prediction of the bore speed propagation. The authors recommend the use of narrow slots to simulate bore propagation in extreme inflow conditions. To alleviate the expected post-shock oscillations that appear behind pipe-filling bores in shock-capturing models, the authors recommend the use of numerical filters, as exemplified in this work.

This work also presented a new approach to handle the flow regime transition in sewers, named Decoupled Pressure Approach. By separately treating the hydrostatic-like pressure and the surcharge pressure terms, this approach overcome a long-known limitation of Preissmann slot models, which is the simulation of low-piezometric head flows. Preissmann slot models regenerate free-surface flows in regions with low piezometric head, even if no ventilation is present. The DPA model has been proposed very recently, but initial tests showed its capacity to simulate diverse flow conditions and replicate the results from other transient flow models (Vasconcelos and Wright, 2005b).

Finally, recent model developments have been successful in including some of the interactions that air and water phases have during rapid filling pipe events. As long as the air pressurization is not so extreme, the interactions between the phases can be handled in a one-dimensional, single phase model framework in which air phase is handled in a simplified fashion. Two-phase flow models based on the shallow water equations are an alternative to be considered when the air pocket length is significant and some of the simplified assumptions are not applicable. The current challenge is to handle the cases when air pressurization becomes significant, and air intrusion on pipe-filling bores occurs. The motion of the air in such cases resembles gravity currents, and models based on the shallow water equations assumptions are no longer applicable due to the high free surface curvature. Future investigations will need to address this problem and other relevant issues such as the geysering through ventilation towers.

\section{References}

Arai, K. and Yamamoto, K. (2003). Transient analysis of mixed free-surface-pressurized flows with modified slot model - part1: Computational model and experiment. Proc. FEDSM03 - 4th ASME-JSME Joint Fluids Engrg. Conference, Honolulu, HW.

ASME/JSME. 
Arora, M. and Roe, P. L. (1997). On post-shock oscillations due to shock capturing schemes in unsteady flows. J. Comp. Physics, 130(1), 25-40.

Cardle, J. A. and Song, C. S. S. (1988). Mathematical modeling of unsteady flow in storm sewers. Int. J. Engrg. Fluid Mech., 1(4), 495-518.

Cunge, J. A., Jr., F. M. H., and Verwey, A. (1980). Practical Aspects of Computational River Hydraulics. Pitman Publishing Ltd., London, UK. ISBN: 0273084429

Cunge, J.A., and Wegner, M., 1964, Integration numerique des equations d'ecoulement de Barre de St. Venant par un schema implicite de differences finies. Application au cas d'une galerie tantot en charge tantot a surface libre. La Houille Blanche, no. 1, p. 33-39 (in French)

DeHenau, V. and Raithby, G. D. (1995). Transient two-fluid model for the simulation of slug flow in pipelines. Int. J. Multiphase Flow, 21(3), 335-349.

DHI Software (2003) Mouse Pipe Flow - Reference Manual, April 2003, Depts. of Civil Engineering and Computer Science, University of Illinois at Urbana-Champaign, 134 pp.

Environmental Protection Agency (2004). Storm Water Management Model - User's Manual - Version 5.0. November 2004, 245 pp.

Garcia-Navarro, P., Priestley, A., and Alcrudo, F. (1994). Implicit method for water flow modeling in channels and pipes. J. Hydr. Res., 32(5), 721-742.

Glaister, P. (1988). Approximate Riemann solutions of the shallow water equations. J. Hydr. Res., 26(3), 293-306.

Guo, Q. and Song, C. S. S. (1990). Surging in urban storm drainage systems. J. Hydr. Engrg., 116(12), 1523-1537.

Hamam, M. A. and McCorquodale, J. A. (1982). Modeling mixed flow in storm sewers. Can. J. Civ. Engrg., (9), 189-196.

Issa, R. I. and Kempf, M. H. W. (2003). Simulation of slug flow in horizontal and nearly horizontal pipes with the two-fluid model. Int. J. Multiphase Flow,29(1), 69-95.

Katopodes N. D. (2003) - Unpublished manuscript

Li, J. and McCorquodale, A. (1999). Modeling mixed flow in storm sewers. J. Hydr. Engrg., 125(11), 1170-1180.

Nguyen, T. D. (1999). Numerical simulation of the flow in a conduit in the presence of a confined air cushion. Int. J. Num. Meth. in Fluids, 29(4), 485-498.

Press, W. H., Flannery, B. P., Teukolsky, S. A., and Vetterling, W. T. (1989). Numerical Recipes in Pascal. Cambridge University Press. ISBN: 0521375169

Roe, P. L. (1981). Approximate Riemann solvers, parameter vectors, and difference schemes. J. Comp. Physics, 43, 357-372.

Song, C. S. S., Cardle, J. A., and Leung, K. S. (1983). Transient mixed-flow models for storm sewers. J. Hydr. Engrg., 109(11), 1487-1504.

Sturm T. W. (2000) Open Channel Hydraulics McGraw-Hill, ISBN: 0070624453, 512 pp.

Toro, E. F. (2001). Shock-Capturing Methods for Free-Surface Shallow Flows. John Wiley and Sons, ISBN: 0471987662, 326 pp.

Trajkovic, B., Ivetic, M., Calomino, F., and DIppolito, A. (1999). Investigation of transition from free surface to pressurized flow in a circular pipe Water Sci. and Tech., 39(9), 105-112. 
Vasconcelos, J. G. and Wright, S. J. (2003a). Laboratory investigation of surges formed during rapid filling of stormwater storage tunnels. Proc. XXX IAHR Congress, Thessaloniki, Greece. IAHR.

Vasconcelos, J. and S.J. Wright. 2004. "Numerical Modeling of the Transition between Free Surface and Pressurized Flow in Storm Sewers." Journal of Water Management Modeling R220-10. doi: 10.14796/JWMM.R220-10.

Vasconcelos, J. and S.J. Wright. 2005a. "Applications and Limitations of Single-Phase Models to the Description of the Rapid Filling Pipe Problem." Journal of Water Management Modeling R223-19. doi: 10.14796/JWMM.R223-19.

Vasconcelos, J. G. and Wright, S. J. (2005b). Comparison between the Decoupled Pressure Approach and current transient flow solvers. Submitted to the Journal of Hydraulic Research - IAHR.

Vasconcelos, J. G., Wright, S. J. and Roe, P. L. (2005). Decoupled pressure approach for the simulation of flow regime transition in sewers Submitted to the Journal of Hydraulic Engineering - ASCE.

Wiggert, D. C. (1972). Transient flow in free-surface, pressurized systems. J. Hydr. Div., 98(HY1), 11-27.

Wylie, E. B. and Streeter, V. L. (1993). Fluid Transients in Systems. Prentice Hall, Upper Saddle River, NJ, ISBN: 0133221733.

Zhou, F, Hicks, F. E., and Steffler, P. M. (2002). Transient flow in a rapidly filling horizontal pipe containing trapped air. J. Hydr. Engrg., 128(6), 625-634. 
